\title{
Evaluation of the Effectiveness and Safety of Alirocumab Use in Statin-Intolerant Veterans
}

\author{
Fiona I. Imarhia, PharmD, BCPS; Elisabeth M. Sulaica, PharmD, BCCP; Tyler Varisco, PharmD; \\ and Marcy Pilate, PharmD, MS, BCPS, BCACP
}

Background: Since 1987, statin therapy has been the mainstay of treatment for hypercholesterolemia, and current practice guidelines recommend statins as first-line therapy given demonstrated reductions in elevated low-density lipoprotein cholesterol (LDL-C) and cardiovascular (CV) mortality reduction in robust clinical trials. The primary objective of this case series was to assess LDL-C reduction associated with alirocumab use in statin-intolerant veterans at a single US Department of Veterans Affairs medical center. The secondary objective was to assess the incidence of CV events.

Methods: A retrospective chart review was conducted to identify statin-intolerant veterans who were initiated on treatment with alirocumab for LDL-C and/or CV risk reduction between June 2017 and May 2019. Electronic health records of all eligible patients who received alirocumab were reviewed, and basic demographics (patient age, sex, and race/ethnicity) as well as medical characteristics at baseline were collected. Baseline LDL-C was compared with levels at 4 and 24 weeks.

Results: Although a majority of patients saw a significant and clinically meaningful reduction in LDL-C, the group of patients with an increase in LDL-C may have benefitted from targeted intervention to improve medication and dietary adherence.

Conclusions: Alirocumab use in 24 statin-intolerant veterans resulted in a significant reduction in LDL-C at 4 and 24 weeks after initiation. Large long-term studies would better evaluate CV benefit associated with alirocumab therapy in a veteran population.
Author affiliations can be found at the end of the article.

Correspondence:

Fiona Imarhia

(fiona.imarhia@va.gov)

Fed Pract. 2021;38(suppl 4). Published online

November 12.

doi:10.12788/fp.0176 n 2016, 17.6 million deaths occurred globally due to cardiovascular disease (CVD) with coronary artery disease (CAD) and ischemic stroke as top contributors. ${ }^{1}$ Elevated lowdensity lipoprotein cholesterol (LDL-C) has been linked to greater risk of atherosclerotic cardiovascular disease (ASCVD); therefore, LDL-C reduction is imperative to decrease risk of cardiovascular (CV) morbidity and mortality. ${ }^{2}$ Since 1987, statin therapy has been the mainstay of treatment for hypercholesterolemia, and current practice guidelines recommend statins as first-line therapy given demonstrated reductions in LDL-C and CV mortality reduction in robust clinical trials. ${ }^{2-4}$ Although generally safe and well tolerated, muscle-related adverse events (AEs) limit optimal use of statins in up to $20 \%$ of individuals who have an indication for statin therapy. ${ }^{5}$ As a consequence, these patients receive suboptimal statin doses or no statin therapy and are at a higher risk for ASCVD. ${ }^{5}$

Proprotein convertase subtilisin/kexin type 9 (PCSK9) inhibitors have been shown to significantly lower LDL-C when used as monotherapy or in combination with statins and/or other lipidlowering therapies. ${ }^{5}$ These agents are currently approved by the US Food and Drug Administration as an adjunct to diet with or without other lipid-lowering therapies for the management of primary hypercholesterolemia (including heterozygous familial hypercholesterolemia), homozygous familial hypercholesterolemia (evolocumab only), and for use in patients with established CVD unable to achieve their lipid-lowering goals with maximally tolerated statin doses and ezetimibe. ${ }^{4}$ With the ability to reduce LDL-C by up to $65 \%$, PCSK9 inhibitors offer an alternative option for LDL-C and potentially CV risk reduction in statin-intolerant patients. ${ }^{5}$

Alirocumab, the formulary preferred PCSK9 inhibitor at the Michael E. DeBakey Veterans Affairs Medical Center (MEDVAMC) in Houston, Texas, has been increasingly used in high-risk statin-intolerant veterans. The primary objective of this case series was to assess LDL-C reduction associated with alirocumab use in statin-intolerant veterans at the MEDVAMC. The secondary objective was to assess the incidence of CV events. This study was approved by the MEDVAMC Quality Assurance and Regulatory Affairs committee.

\section{METHODS}

In this single-center case series, a retrospective chart review was conducted to identify statin-intolerant veterans who were initiated on treatment with alirocumab for LDL-C and/ or CV risk reduction between June 2017 and May 2019. Adult veterans with a diagnosis of primary hypercholesterolemia (including heterozygous familial hypercholesterolemia) and/or CAD with documented statin intolerance were included in the study. Statin intolerance was defined in accordance with the National Lipid Association (NLA) definition as 
aninability to tolerate $\geq 2$ statins with a trial of at least 1 statin at its lowest daily dose. ${ }^{5}$ Veterans who previously received treatment with evolocumab, those prescribed concurrent statin therapies, and those missing follow-up lipid panels at 24 weeks were excluded from the study. To assess LDL-C reduction, LDL-C at baseline was compared with LDL-C at 4 and 24 weeks. Incident $\mathrm{CV}$ events before and after alirocumab initiation were documented. The US Department of Veteran Affairs (VA) Computerized Patient Record System was used to collect patient data.

\section{Data Collection, Measures, and Analysis}

Electronic health records of all eligible patients who received alirocumab were reviewed, and basic demographics (patient age, sex, and race/ ethnicity) as well as medical characteristics at baseline were collected. To confirm statin intolerance, each veteran's history of statin use and use of additional lipid-lowering agents was documented. CV history was measured with an index of categorical measures for hypertension, confirmed CAD, hyperlipidemia, heart failure, arrhythmias, peripheral artery disease, stroke, diabetes mellitus, and hypothyroidism. Additionally, concomitant medications, such as aspirin, $P 2 Y_{12}$ inhibitors, $\beta$-blockers, angiotensin-converting enzyme inhibitors, and angiotensin II receptor blockers that patients were taking also were collected. Each veteran's lipid panel at baseline, and at 4 and 24 weeks posttreatment initiation, also was extracted. Continuous variables were summarized with means (SD), and categorical variables were summarized with frequencies and proportions. The paired Wilcoxon signed rank test was used to compare LDL-C at 4 and 24 weeks after alirocumab initiation with patients' baseline LDL-C.

\section{RESULTS}

Between June 2017 and May 2019, 122 veterans were initiated on alirocumab. Of these veterans, 98 were excluded: 35 concurrently received statin therapy, 33 missed follow-up lipid panels, 21 had previously received evolocumab, 6 failed to meet the NLA definition for statin intolerance, 2 did not fill active alirocumab prescriptions, and 1 had an incalculable LDL-C with a baseline triglyceride level of $3079 \mathrm{mg} / \mathrm{dL}$. This resulted in 24 veterans included in the analysis.

Most participants were male (87.5\%) and
TABLE Baseline Characteristics ( $\mathrm{N}=24)$

\begin{tabular}{|c|c|}
\hline Characteristics & Results \\
\hline Male, No. (\%) & $21(87.5)$ \\
\hline Age, mean (SD), y & $66.0(8.4)$ \\
\hline LDL-C, mean (SD), mg/dL & $161.9(74.3)$ \\
\hline \multicolumn{2}{|l|}{ Race/ethnicity, No. (\%) } \\
\hline White & $19(79.2)$ \\
\hline Black & $3(12.5)$ \\
\hline Hispanic or Latino & $2(8.3)$ \\
\hline \multicolumn{2}{|l|}{ Prior statin use history, No. (\%) } \\
\hline Atorvastatin & $23(95.8)$ \\
\hline Simvastatin & $19(79.2)$ \\
\hline Rosuvastatin & $19(79.2)$ \\
\hline Pravastatin & $15(62.5)$ \\
\hline Other & $8(33.3)$ \\
\hline \multicolumn{2}{|l|}{ Other lipid-lowering therapies, No. (\%) } \\
\hline Ezetimibe & $8(33.3)$ \\
\hline Omega-3 fatty acid & 7 (29.2) \\
\hline Niacin & $2(8.3)$ \\
\hline Other agents & $4(16.7)$ \\
\hline \multicolumn{2}{|l|}{ Cardiovascular history, No. (\%) } \\
\hline Hyperlipidemia & $21(87.5)$ \\
\hline Coronary artery disease & $19(79.2)$ \\
\hline Previous coronary artery bypass surgery & $11(57.9)$ \\
\hline Previous percutaneous coronary intervention & $6(31.6)$ \\
\hline Stable angina & $3(15.8)$ \\
\hline Hypertension & $17(70.8)$ \\
\hline Diabetes mellitus & $9(37.5)$ \\
\hline Hypothyroidism & $4(16.7)$ \\
\hline Heart failure & $3(12.5)$ \\
\hline Familial hypercholesterolemia & $2(8.3)$ \\
\hline Stroke & $2(8.3)$ \\
\hline Arrhythmias & $2(8.3)$ \\
\hline Peripheral artery disease & $2(8.3)$ \\
\hline \multicolumn{2}{|l|}{ Other cardiovascular medications, No. (\%) } \\
\hline Aspirin & $14(58.3)$ \\
\hline$\beta$-blocker & $10(41.7)$ \\
\hline Angiotensin receptor blockers & $8(33.3)$ \\
\hline Clopidogrel & $6(25.0)$ \\
\hline Prasugrel & $1(4.2)$ \\
\hline
\end{tabular}

Abbreviation: LDL-C, low-density lipoprotein cholesterol.

White veterans $(79.2 \%)$ with a mean (SD) age of 66.0 (8.4) years and mean (SD) baseline LDL-C of 161.9 (74.3) mg/dL. At baseline, 21 veterans had a history of primary hyperlipidemia, 19 had a history of CAD, and 2 had a history of heterozygous familial hypercholesterolemia. Of the 24 patients included, the most trialed statins before alirocumab initiation were atorvastatin (95.8\%), simvastatin $(79.2 \%)$, rosuvastatin $(79.2 \%)$, and pravastatin (62.5\%) (Table). 


\section{LDL-C Reduction}

Veterans were initially treated with alirocumab $75 \mathrm{mg}$ administered subcutaneously every 2 weeks; however, 11 veterans required a dose increase to $150 \mathrm{mg}$ every 2 weeks. At treatment week 4 , the median LDL-C reduction was $78.5 \mathrm{mg} / \mathrm{dL}$ (IQR, 28.0-107.3; $P<.01$ ), and at treatment week 24 , the median LDL-C reduction was $55.6 \mathrm{mg} / \mathrm{dL}$ (IQR, 18.6-85.3; $P<.01)$. This equated to median LDL-C reductions from baseline of $48.5 \%$ at week 4 and $34.3 \%$ at week 24 . A total of 3 veterans experienced LDL-C increases following initiation of alirocumab. At week 4, 9 veterans were noted to have an LDL-C reduction > 50\%, 7 veterans had an LDL-C reduction between $30 \%$ and $50 \%$, and 5 veterans had an LDL-C reduction of $<30 \%$. At week 24, 6 had an LDL-C reduction $>50 \%$, 9 veterans had an LDL-C reduction between $30 \%$ and $50 \%$, and 6 had a LDL-C reduction $<30 \%$.

\section{Cardiovascular Events}

Before alirocumab initiation, $22 \mathrm{CV}$ events and interventions were reported in 16 veterans: 12 percutaneous coronary interventions, 5 coronary artery bypass surgeries (CABG), 4 myocardial infarctions, and 1 transient ischemic attack. One month following alirocumab initiation, 1 veteran underwent a CABG after a nonST-elevation myocardial infarction (NSTEMI).

\section{Safety and Tolerability}

Alirocumab was discontinued in 5 veterans due to 4 cases of intolerance (reported memory loss, lethargy, myalgias, and body aches with dyspnea) and 1 case of persistent LDL-C of $<40 \mathrm{mg} / \mathrm{dL}$. Alirocumab was discontinued after 1 year in 2 patients (persistent LDL-C $<40 \mathrm{mg} / \mathrm{dL}$ and reported memory loss) and after 6 months in the veteran who reported lethargy. Alirocumab was discontinued after 4 months in the veteran with myalgias and within 2 months in the veteran with body aches and dyspnea. No other AEs were reported.

\section{DISCUSSION}

The Efficacy and Safety of Alirocumab vs Ezetimibe in Statin-Intolerant Veterans With a Statin Rechallenge Arm trial is the first clinical trial to examine the efficacy and safety of alirocumab use in statin-intolerant patients. In the trial,
314 patients were randomized to receive alirocumab, ezetimibe, or an atorvastatin rechallenge. ${ }^{6}$ At 24 weeks, alirocumab reduced mean (SE) LDL-C by $45.0 \%$ (2.2\%) vs $14.6 \%$ (2.2\%) with ezetimibe (mean difference $30.4 \%$ [3.1\%], $P<.01) .{ }^{6}$ Fewer skeletal-muscle-related events also were noted with alirocumab vs atorvastatin (hazard ratio, $0.61 ; 95 \% \mathrm{Cl}, 0.38-0.99 ; P=.04$ ). ${ }^{6}$

In this case series, an LDL-C reduction of $>50 \%$ was observed in 9 veterans $(42.9 \%)$ following 4 weeks of treatment; however, LDL-C reduction of $>50 \%$ compared with baseline was sustained in only 6 veterans $(28.6 \%)$ at week 24. Additionally, LDL-C increases from baseline were observed in 3 veterans; the reasoning for the observed increase was unclear, but this may have been due to nonadherence and dietary factors. ${ }^{4}$ Although a majority of patients saw a significant and clinically meaningful reduction in LDL-C, the group of patients with an increase in the same may have benefitted from targeted intervention to improve medication and dietary adherence. PCSK9 inhibitor resistance also may have contributed to an increase in LDL-C during treatment. ${ }^{7}$

Of the 24 patients included, 4 reported AEs resulted in therapy discontinuation. Memory impairment, a rare $A E$ of alirocumab, was reported 1 year following alirocumab initiation. Additionally, lethargy was reported after 6 months of treatment. Myalgia also was reported in a veteran 4 months following treatment, and 1 veteran experienced body aches and dyspnea $<2$ months following treatment. The most common AEs associated with alirocumab, as noted in previous safety and efficacy clinical trials, included: nasopharyngitis, injection site reaction, influenza, urinary tract infection, and myalgias. ${ }^{8}$ Many of these more common AEs may be subclinical and underreported. This small case series, however, detected 4 events severe enough to lead to therapy discontinuation. Although this sample is not representative of all statin-intolerant patients who receive treatment with alirocumab, our findings suggest the need for patient education on potential AEs before therapy initiation and clinician monitoring at follow-up visits.

The ODYSSEY OUTCOMES trial established a CV benefit associated with alirocumab; however, patients included had a recent acute coronary syndrome event and were receiving a high-intensity statin. ${ }^{9}$ This case series is unique in that before alirocumab 
initiation, $22 \mathrm{CV}$ events/interventions were reported in the sample of 24 patients. After therapy initiation, 1 patient underwent a CABG after an NSTEMI in the month following initiation. This suggests that cardiac complications are possible after PCSK-9 initiation; however, little information can be gained from 1 patient. Nevertheless, early therapy failure should be investigated in the context of real-world use in statin-intolerant patients. This is a complex task, however, given the difficulties of achieving a balanced study design. Statin intolerance is a clear source of selection bias into treatment with alirocumab as patients in this population have already initiated and failed statin therapy. The prevalence of prior CV events and the time-dependent association between prior and future $\mathrm{CV}$ events stand as another complex confounder. Although there is a clear and pressing need to understand the risks and benefits of PCSK9 therapy in statin-intolerant patients, future research in this area will need to cautiously address these important sources of bias.

Overall, the results of this case series support LDL-C reduction associated with alirocumab in the absence of statin therapy. Despite favorable results, use of alirocumab may be limited by cost and its subcutaneous route of administration. Bempedoic acid, an oral, once-daily lipidlowering agent poses an alternative to PCSK9 inhibitors, but further data regarding CV outcomes with this agent is needed. ${ }^{10,11}$ Robust randomized controlled trials also are needed to evaluate $\mathrm{CV}$ outcomes for alirocumab use in statin-intolerant veterans.

\section{Limitations}

Only 24 veterans were included in the study, reflecting $20 \%$ of the charts reviewed $(80 \%$ exclusion rate), and in this small sample, only $1 \mathrm{CV}$ event was observed. Both of these serve as threats to external validity. As the study information was extracted from chart review, the results may be limited by coding or historical bias. Medical information from outside institutions may be missing from medical records. Additionally, results may be skewed by possible documentation errors. Furthermore, the period between previous $\mathrm{CV}$ events and alirocumab initiation is unclear as event dates were often not recorded if treatment was received at an outside institution.
Due to the short follow-up period, the case series is limited in its assessment of CV outcomes and safety outcomes. Larger studies over an extended period are needed to assess CV outcomes and safety of alirocumab use in statin-intolerant patients. Also, medication adherence was not assessed. Given the impact of medication adherence on LDL-C reduction, it is unclear what role medication adherence played in the LDL-C reduction observed in this study. ${ }^{4}$

\section{CONCLUSIONS}

Alirocumab use in 24 statin-intolerant veterans resulted in a significant reduction in LDL-C at 4 and 24 weeks after initiation. In addition, $1 \mathrm{CV}$ event/intervention was observed following alirocumab initiation, although this should be interpreted with caution due to the retrospective nature of this case series, small sample size, and short follow-up period. Large, long-term studies would better evaluate the $\mathrm{CV}$ benefit associated with alirocumab therapy in a veteran population.

\section{Author affiliations}

Fiona Imarhia is a Clinical Pharmacy Specialist at Michael E. DeBakey Veteran Affairs Medical Center in Houston, Texas. Elisabeth Sulaica is a Clinical Assistant Professor in the Department of Pharmacy Practice and Translational Research, and Tyler Varisco is a Research Assistant Professor in the Department of Pharmaceutical Health Outcomes and Policy, both at the University of Houston College of Pharmacy. Marcy Pilate is an Inpatient Pharmacy Supervisor at G.V. (Sonny) Montgomery Veterans Affairs Medical Center in Jackson, Mississippi.

\section{Author disclosures}

The authors report no actual or potential conflicts of interest with regard to this article.

\section{Disclaimer}

The opinions expressed herein are those of the authors and do not necessarily reflect those of Federal Practitioner, Frontline Medical Communications Inc., the US Government, or any of its agencies. This article may discuss unlabeled or investigational use of certain drugs. Please review the complete prescribing information for specific drugs or drug combinations-including indications, contraindications, warnings, and adverse effects-before administering pharmacologic therapy to patients.

\section{References}

1. Benjamin EJ, Munter P, Alonso A, et al; American Heart Association Council on Epidemiology and Prevention Statistics Committee and Stroke Statistics Subcommittee. Heart disease and stroke statistics - 2019 update: a report from the American Heart Association. Circulation. 2019;139(10):e56-e528. doi:10.1161/CIR.0000000000000659

2. Stone NJ, Robinson JG, Lichtenstein AH, et al; American College of Cardiology/American Heart Association Task Force on Practice Guidelines. 2013 ACC/AHA guideline on the treatment of blood cholesterol to reduce atherosclerotic cardiovascular risk in adults: a report of the American College of Cardiology/American Heart Association 
Task Force on Practice Guidelines. Circulation. 2014 Jun 24;129(25)(suppl 2):S1-S45. doi:10.1016/j.jacc.2013.11.002

3. Hajar R. Statins: past and present. Heart Views. 2011;12(3): 121-127. doi:10.4103/1995-705X.95070

4. Grundy SM, Stone NJ, Bailey AL, et al. 2018 AHA/ACC/ AACVPR/AAPA/ABC/ACPM/ADA/AGS/APhA/ASPC/ NLA/PCNA guideline on the management of blood cholesterol: a report of the American College of Cardiology/ American Heart Association Task Force on Clinical Practice Guidelines. J Am Coll Cardiol 2019;73(4):3168-3209. doi:10.1016/j.jacc.2018.11.002

5. Toth PH, Patti AM, Giglio RV, et al. Management of statin intolerance in 2018: still more questions than answers. Am J Cardiovasc Drugs. 2018;18(3):157-173. doi:10.1007/s40256-017-0259-7

6. Moriarty PM, Thompson PD, Cannon CP, et al; ODYSSEY ALTERNATIVE Investigators. Efficacy and safety of alirocumab vs ezetimibe in statin-intolerant patients, with a statin rechallenge arm: The ODYSSEY ALTERNA-
TIVE randomized trial. J Clin Lipidol. 2015;9(6):758-769. doi:10.1016/j.jacl.2015.08.006

7. Shapiro MD, Miles J, Tavori H, Fazio S. Diagnosing resistance to a proprotein convertase subtilisin/kexin type 9 inhibitor. Ann Intern Med. 2018;168(5):376-379. doi:10.7326/M17-2485

8. Raedler LA. Praluent (alirocumab): first PCSK9 inhibitor approved by the FDA for hypercholesterolemia. Am Health Drug Benefits. 2016;9:123-126.

9. Schwartz GC, Steg PC, Szarek M, et al; ODYSSEY OUTCOMES Committees and Investigators. Alirocumab and cardiovascular outcomes after acute coronary syndrome. N Engl J Med. 2018;379(22):2097-2107. doi:10.1056/NEJMoa1801174

10. Nexletol. Package insert. Esperion Therapeutics Inc; 2020.

11. Laufs U, Banach M, Mancini GBJ, et al. Efficacy and safety of bempedoic acid in patients with hypercholesterolemia and statin intolerance. J Am Heart Assoc. 2019;8(7):e011662. doi:10.1161/JAHA.118.011662 